\title{
Low Doses of Imidacloprid Induce Oxidative Stress and Neural Cell Disruption in Earthworm Eisenia fetida
}

\author{
Artem Huslystyi $i^{1, a}$, Victor Nedzvetsky ${ }^{1,2, b}$, Serhii Yermolenko ${ }^{1, c}$, \\ Viktor Gasso ${ }^{1, \mathrm{~d}, ~}{ }^{*}$, Vlad Petrushevskyi ${ }^{1, \mathrm{e}}$ and Elena Sukharenko ${ }^{3, \mathrm{f}}$
}

${ }^{1}$ Research Institute of Biology, Oles Honchar Dnipro National University, Dnipro, Ukraine

${ }^{2}$ Bingöl University, Bingöl, Turkey

${ }^{3}$ Kerch State Maritime Technological University, Kerch, Ukraine

aguslistyj@i.ua, bnedzvetskyvictor@ukr.net, cserejamahno@gmail.com, dviktor.gasso@gmail.com, evlad.petrushevskiy@gmail.com, fhelenasuhar@gmail.com

Keywords: neonicotinoid, neuron-specific enolase, NSE, reactive oxygen species, ROS, lipid peroxidation, LPO.

\begin{abstract}
Imidacloprid is a widely used pesticide that belongs to the class of neonicotinoids. There is a piece of rising evidence that neonicotinoids exert cytotoxic effects in non-target organisms including vertebrate species such as mammals. Nevertheless, dose-limiting toxicity and molecular mechanisms of neonicotinoids' deleterious effects are still poorly understood. In accord to imidacloprid fate in the environment, the most of used pesticide is absorbed in the soil. Therefore, earthworms, which are prevailing soil organisms, could be considered as a target of neonicotinoids toxicity. The earthworm's simple nervous system is a prospective model for neurotoxicological studies. We exposed earthworms to imidacloprid in a paper contact test with a doses range of $0.1-0.4 \mu \mathrm{g} / \mathrm{cm}^{2}$ for 14 days. In the present work, we studied the imidacloprid effect on oxidative stress generation and neuronal marker neuron-specific enolase (NSE) expression. The exposure to imidacloprid induced a dose-dependent decrease in NSE. Both reactive oxygen species production and lipid peroxidation level were upregulated as well. Observed NSE decline suggests imidacloprid-caused disturbance in earthworm neuron cells. Obtained data have shown that relatively low doses of imidacloprid are potent to induce cytotoxicity in neurons. Furthermore, neurotoxicity could be recognized as one of an individual scenario of the general imidacloprid toxicity. Thus, presented results suggest the cytotoxicity of imidacloprid low doses in non-target organisms and hypothesize that NSE downregulation could be estimated as a biomarker of neonicotinoid cytotoxicity in a nervous system of non-insect species.
\end{abstract}

\section{Introduction}

Imidacloprid [(2E)-1-((6-chloro-3-pyridinyl) methyl)-N-nitro-2-imidazolidinimine] is one of the most often used neonicotinoid insecticides worldwide owing to its anti-insect efficacy and primary thought of being inoffensive to mammals [1,2]. Neonicotinoids are a relatively novel class of insecticides designed in the last decades of the $20^{\text {th }}$ century. They form a family of pesticides that are widespread due to their unique mechanism of insect neural system suppression. They act as agonists in the insect nicotinic acetylcholine receptors (nAChR) and their promised low toxicity to vertebrates is determined by their dissimilar target receptor sites [3]. Among other consequences, the imidacloprid exposure resulted in oxidative stress, lipid peroxidation and varying degrees of neuron degeneration that induce multiple neurobehavioral aberrations and neurotoxic impacts [4].

One of the environmental pollution risk factors relates to the established fact that some imidacloprid is potent to keep their toxicity in soil up to several years [5,6]. Uncontrolled and extensive use of imidacloprid is one of the important causes of global contamination of the environment. There was demonstrated that the accumulation of imidacloprid in a soil is prevailing among other neonicotinoids as well as its migration rate into the soil profiles [7]. Imidacloprid are able to move vertically and horizontally in soils and to decline non-target soil organisms [8]. Hence, continuous agricultural application of imidacloprid is a critical cause of significant pollution and 
permanent intoxication of non-target organisms including animals and humans. The study of the deleterious effects of imidacloprid under current conditions of its application is actual and extremely important. Taking into account that imidacloprid affects neuronal cells as a first target, the abnormalities of neural system could be an important component of its multiple toxicity.

Earthworms are widespread soil organisms, which affected with imidacloprid through the consuming soil particles and plant remains in treated areas. On the other hand, earthworms are crucial organisms to maintain the soil system stability. Earthworms provide key soil functions entailed in important ecosystem services that are substantial for agroecosystem sustainability. They produce organic matter and stabilize the soil organic matter, enlarge levels of nutrients (nitrogen, phosphorous, and potassium), enhance soil nutrient cycling and activity of beneficial soil microorganisms. [9] Oxidative stress generation and DNA damage were proved as most prominent consequences of imidacloprid toxicity in non-target species $[10,11]$. Recent studies have shown that earthworms are susceptible to insecticides toxicity. Insecticide exposure leads to decline in earthworm population and soil biodiversity [12]. Furthermore, neonicotinoids in general and imidacloprid in particular can disrupt enzymatic activities, increase individual mortality, decrease fecundity and growth, change feeding rate and decrease the overall community biomass and population density of earthworms [13]. Besides, there is a meaningful knowledge gap in respect to data on the imidacloprid toxicity to soil invertebrate biota and the earthworms could be adequate objects to study both molecular mechanisms and toxic effects of neonicotinoid contamination.

\section{Theory}

A global neonicotinoid pollution of the environment is one of the critical problems in the whole world for the last decades. This class of systemic insecticides was designed as a tool against chewing and sucking insects mainly. Despite the first aim of neonicotinoids to kill the insect pests and $\mathrm{nAChR}$ is the main target of neuronal specificity, there is growing evidence of the toxicity risk for other non-target species. Several reports have shown that neonicotinoids including imidacloprid are potent to initiate a large number of disturbances caused by multiple cytotoxic effects [14-16].

There is a piece of growing evidence that imidacloprid and other neonicotinoids exert multiple toxic effects in different living organisms including earthworms [17-20]. These deleterious effects are accompanied by metabolic disorders, reactive oxygen species (ROS) production, and suppression of antioxidant systems in earthworms [14, 21, 22]. Oxidative stress launches a set of negative molecular processes [23, 24]. The harmful effect of imidacloprid exposure was determined in the worm Caenorhabditis elegans (Maupas, 1900), where the abnormality of growth, disruption of locomotion, and fertility were detected [15]. Recently, there was reported that imidacloprid can induce sperm deformity and DNA damage in Eisenia fetida (Savigny, 1826) in a low dose $(500 \mu \mathrm{g} / \mathrm{kg})$ in the contaminated soil [25]. Exposure to other neonicotinoid dinotefuran affects redox balance inhibits antioxidative enzymatic activity and functional gene expression in earthworms E. fetida [26]. However, limited information is available on the molecular mechanism of neonicotinoid toxicity to earthworms.

Some authors have emphasized the disruption of vital functions in fish and mammals with acute neonicotinoid toxicity $[16,27]$. However, owing to permanent existing of imidacloprid in the soil it is imperative to examine its dose-dependent cytotoxicity in soil organisms. Cell culture models have shown the prominent toxicity of imidacloprid in respect to neurogenesis and neural tube cells viability [28]. Other authors demonstrated the effects of imidacloprid on the basic metabolic pathways. There was shown that imidacloprid induces lipid accumulation in mammal adipocyte differentiated 3T3-L1 cells [29]; it can affect intestine barrier function [30]. Additionally, imidacloprid affects glucose metabolism through inhibition of protein kinases phosphorylation that consequently suppresses insulin signaling and initiates insulin resistance [31].

Recent studies are focused on neural tissue cells disturbances. Similar to insect abnormalities of nicotinic acetylcholine receptors were detected in various organisms including rats and birds [32, 33]. Furthermore, the detrimental effect of imidacloprid was most meaningful in adolescent rat brains in comparison with the adult ones. Another study has shown imidacloprid-caused 
neurobehavioral deficits in offspring rats initiated with in utero exposure where critical changes in glial intermediate filaments occur in the hippocampus and the motor cortex [32]. Therefore, imidacloprid is potent to provoke plural disturbances, which are associated with their direct effect on nicotinic acetylcholine receptors.

Despite well-studied direct imidacloprid toxicity for insects, the real toxicity for the earthworms could be supplemented with the indirect effects on neuronal cells and its viability. Recent results have shown the impairment of earthworm metabolomics caused by imidacloprid [21, 22]. The simple nervous system of earthworms may be critically susceptible to neonicotinoids toxicity. The damaging of signaling pathways between the neural system and other organs can cause a lack of cell viability as well as the disruption of vital functions. The searching for tissue-specific molecular biomarkers, which are potent to reflect the cellular damages and a decline in vital functions, could be a fruitful tool of pesticide pollution effects estimation [34-37]. Several neuron-specific proteins are considered as prospective candidates to use for the disruption of the molecular mechanisms in different species $[32,38,39]$. Some of them are confirmed the biomarkers of the neural system injury [40,41], and the modulation of NSE expression could be estimated as a biomarker of both oxidative stress and the disruption of neuronal cell integrity [42]. There is no accessible data on the expression of the NSE in the earthworms' neural cells.

Earthworms have been accepted for assessment of chemical toxicity on soil biota. Earthworm E. fetida was selected as the test species because it is easy-to-use in laboratory experiments and the protocols for them are well developed and adopted [43]. The toxicity test using E. fetida is quite informative for our research goal that is not oriented on ecological significance in a real ecosystem. Taking together, the study of neonicotinoid toxicity is actual and important to elucidate molecular mechanisms of its detrimental effect. To address the aforementioned knowledge gaps, earthworms E. fetida were exposed to imidacloprid with doses closed to the realistic concentration of imidacloprid to clarify the molecular mechanism of its cytotoxic effect.

The aim of the present work was the study of neurotoxic effect induced by the exposure of earthworms to nature-realistic doses of imidacloprid.

\section{Materials and Methods}

Treatment procedure. Earthworms E. fetida $(0.25 \pm 0.037 \mathrm{~g}$ and $9.7 \pm 0.85 \mathrm{~cm})$ and natural soil substrate were purchased from the LLC "Rich Soil" (Zaporizhia, Ukraine) and delivered in special transportation aerated wood containers to the laboratory. Then the earthworms were placed in wooden boxes with natural soil substrate to acclimatize for 20 days $\left(18 \pm 2{ }^{\circ} \mathrm{C}\right.$, soil humidity $85 \pm 5 \%$ ).

The exposure to imidacloprid was carried out using the filter paper contact test [43]. The earthworms E. fetida were treated with the imidacloprid doses of $0.1,0.2$, and $0.4 \mu \mathrm{g} / \mathrm{cm}^{2}$ of paper. Subsequently, $2.0 \mathrm{~mL}$ of acetone containing the test substance was added to a piece of Whatman filter paper (grade 1) in a 9-cm Petri dish, and $2 \mathrm{~mL}$ of distilled water was added to the filter paper after the acetone was completely evaporated. Each earthworm was rinsed with the physiological solution and placed on the filter paper in a separate Petri dish. Pure acetone was used as the control trial. Each earthworm was individually treated with one of the aforementioned doses under conditions of $20 \pm 1{ }^{\circ} \mathrm{C}$ in the darkness and relative humidity of $80-85 \%$ for 24 hours. After $24 \mathrm{~h}$ treatment, the earthworms were removed into artificial soil in a $500-\mathrm{mL}$ beaker at $20 \pm 1{ }^{\circ} \mathrm{C}$ for $24 \mathrm{~h}$ in the darkness for rehabilitation. The exposure-rehabilitation procedure was repeated for 14 days. Every earthworm was exposed to the respective imidacloprid dose for $7 \times 24$ hours.

Earthworms having empty gut were sacrificed according to the rules adopted by the Bioethics Committee of Oles Honchar Dnipro National University. The whole earthworm tissue was washed out with ice-cold phosphate buffer saline (PBS). Then the PBS was removed with filter paper and the tissue samples were subjected to protein extraction. 
Tissue analysis. Each sample was homogenized on the ice in a 1:15 (w/v) ratio with using lysis buffer $-10 \mathrm{mM}$ Tris-buffer $(\mathrm{pH}=7.4)$ containing $0.1 \mathrm{mM} \mathrm{NaCl}, 1 \%$ TritonX-100, 0.2\% SDS, $2.5 \mathrm{mM}$ ethylenediaminetetraacetic acid (EDTA), $6.5 \mu \mathrm{M}$ aprotinin, $1.5 \mu \mathrm{M}$ pepstatin $\mathrm{A}, 23 \mu \mathrm{M}$ leupeptin, $1 \mathrm{mM}$ phenylmethylsulfonylfluoride (PMSF), $1 \mu \mathrm{M}$ sodium orthovanadate $5 \mu \mathrm{M}$ soybean trypsin inhibitor. After homogenization, obtained suspensions were incubated on the ice for $60 \mathrm{~min}$ to extract the proteins. The homogenates after incubation were centrifuged at $40.000 \mathrm{~g}$ for $45 \mathrm{~min}$ in a cooled centrifuge. The supernatants contained protein extract were sampled into microcentrifuge tubes and stored at $-80^{\circ} \mathrm{C}$ before western blot analysis.

The reactive oxygen species (ROS) level in homogenates was measured by using the fluorometric method based on a 2',7'-dichlorofluorescein diacetate (DCFHDA) reaction. The supernatant $(200 \mu \mathrm{L})$ was mixed with $10 \mu \mathrm{M}$ DCFHDA and incubated for $30 \mathrm{~min}$ at $37^{\circ} \mathrm{C}$. The measuring of ROS levels was performed with a SpectraMax Gemini EM spectrofluorometer with $485 \mathrm{~nm}$ wavelength excitation and $530 \mathrm{~nm}$ emission. Every procedure was performed according to the ROS assay kit producer. Each sample was tested in triply parallels.

The end-products content of lipid peroxidation (LPO), MDA+4-hydroxialkenes, was measured with the absorbance estimation of the complex MDA-thiobarbituric acid by spectrophotometric method at $532 \mathrm{~nm}$. Tetraethoxypropane was used as the standard [44].

Western blot of proteins. To determine the NSE level Western blotting was performed using SDS-PAGE electrophoresis in a $5-20 \%$ gradient of acrylamide. Separated in polyacrylamide gel proteins were transferred with electric field $20 \mathrm{~V} / \mathrm{cm}$ at $120 \mathrm{~min}$ onto polyvinylidenefluoride (PVDF) membrane. The membrane was washed with PBS and incubated in blocking tris-saline buffer (TBST) containing $0.05 \%$ Tween-20 and 3\% bovine serum albumin (BSA) at 60 min. After blocking, the membranes were incubated overnight at $+4{ }^{\circ} \mathrm{C}$ with the primary rabbit monoclonal antibody, anti-NSE (Abcam, ab79757, 1:3000 dilution), an d mouse monoclonal anti-GAPDH (Abcam, ab8226, 1:5000 dilution). After the incubation with primary antibodies, the membrane was triply washed with TBST. Washed membranes were incubated with corresponded anti-rabbit HRPlabeled secondary antibody for $60 \mathrm{~min}$ and were washed with the TBST triply again. The results of western blot were developed with an ECL kit based on the chemiluminescence method with the use of the automatic X-ray machine (Carestream Health Inc., USA) [45].

Densitometric analysis of immunostained NSE polypeptide zones was performed with the use of TotalLab TL120 software (USA). The intensity value obtained with scanning every individual band was normalized to the intensity in respect to the correspondent GAPDH band. Every track obtained in the scanned picture was corrected to the background level, which corresponds to the nonreactive area on the X-ray film.

We carried out analyses in six independent experiments. Statistical comparisons of the data were performed using a one-way analysis of variance (ANOVA) followed by Tukey's post-hoc test using Origin, 2019 (OriginLab Corporation, the USA). Data are expressed as the mean (M) with standard error (SEM) of at least six independent experiments; p-values less than 0.05 were accepted as statistically significant. GraphPad Prism 9 (GraphPad Software, the USA) was used for plots design. 


\section{Results}

To pursue the aim of this study, we carried out the exposure to imidacloprid by its application in a contact toxicity manner. Oxidative stress is confirmed as the common driver of both structural and functional disruption in earthworm organisms. Since pesticides are known as xenobiotics, which potent to induce redox imbalance in various species, we have been measured the main indices of oxidative stress: ROS (Fig. 1) and lipid peroxidation (LPO) levels in the earthworms.

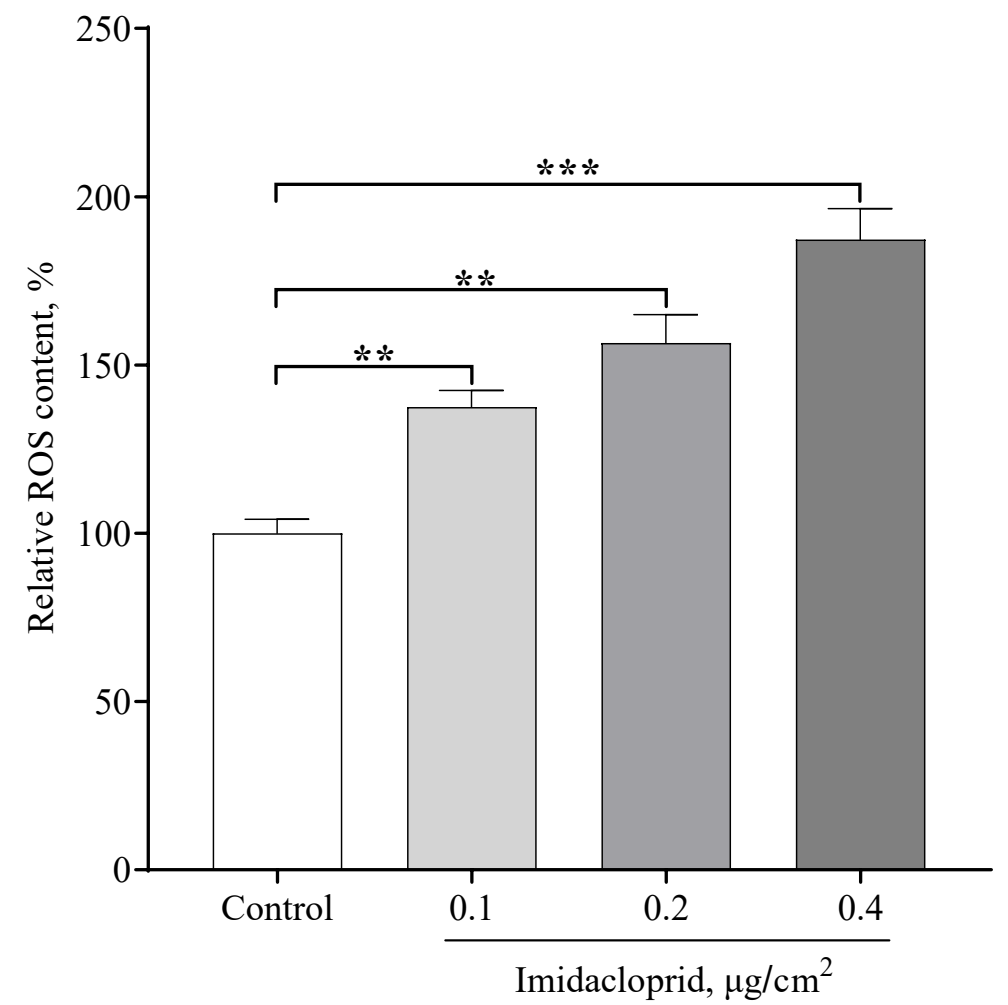

Figure 1. Effects of imidacloprid $\left(0.1,0.2\right.$ and $0.4 \mu \mathrm{g}$ per $1 \mathrm{~cm}^{2}$ of contact paper) on the reactive oxygen species (ROS) production in the treated earthworms; $* * p<0.01, * * * p<0.001$ (compared with the control group).

To investigate the realistic effects of imidacloprid low doses on the ROS level the measuring was carried out in the extracts from the worm whole tissue. A dose-dependent increase in ROS content was observed in the earthworms exposed to imidacloprid in doses of $0.1-0.4 \mu \mathrm{g}$ in comparison to the untreated control animals. Since LPO level is a function of ROS generation and reflects membrane disruption, we also measured this index to estimate oxidative damaging of cellular membranes. A statistically significant increase in LPO level has also been observed in all treated groups of earthworms in comparison with the untreated control (Fig. 2). 


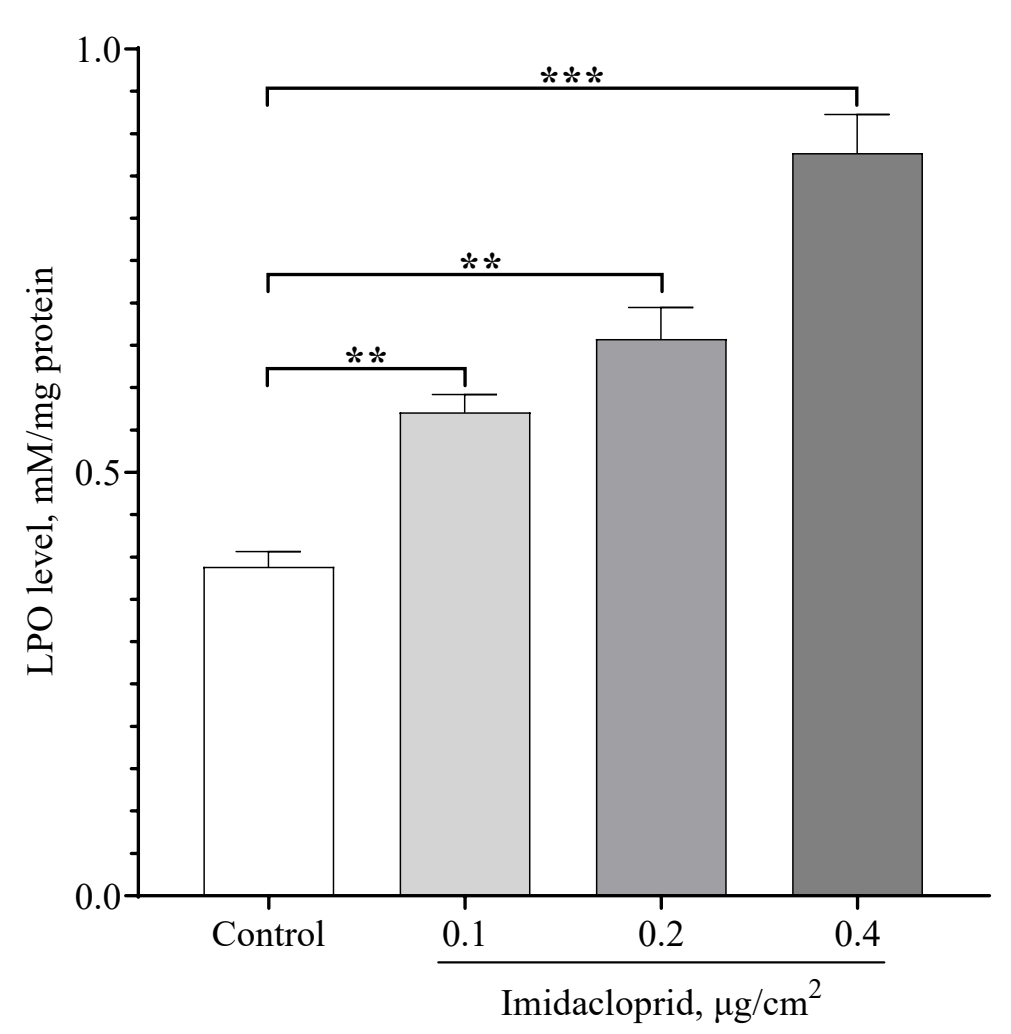

Figure 2. Effects of imidacloprid (0.1, 0.2 and $0.4 \mu \mathrm{g}$ per $1 \mathrm{~cm}^{2}$ of contact paper) on the lipid peroxidation (LPO) in the treated earthworms; $* * p<0.01, * * * p<0.001$

(compared with the control group).

Neuron-specific enolase (NSE) is an acidic protease known for nervous cells as a thoughtful bioindicator of the injury and diagnosis of these cells. That is why we investigated the possible neurotoxic effect of imidacloprid on the NSE expression in earthworms. The progressive decline in NSE expression was observed in earthworm groups exposed to 0.2 and $0.4 \mu \mathrm{g}$ doses (Fig. 3). Besides, there were not detected statistically significant changes in the earthworm group treated with the dose of $0.1 \mu \mathrm{g}$ as compared to the control. 


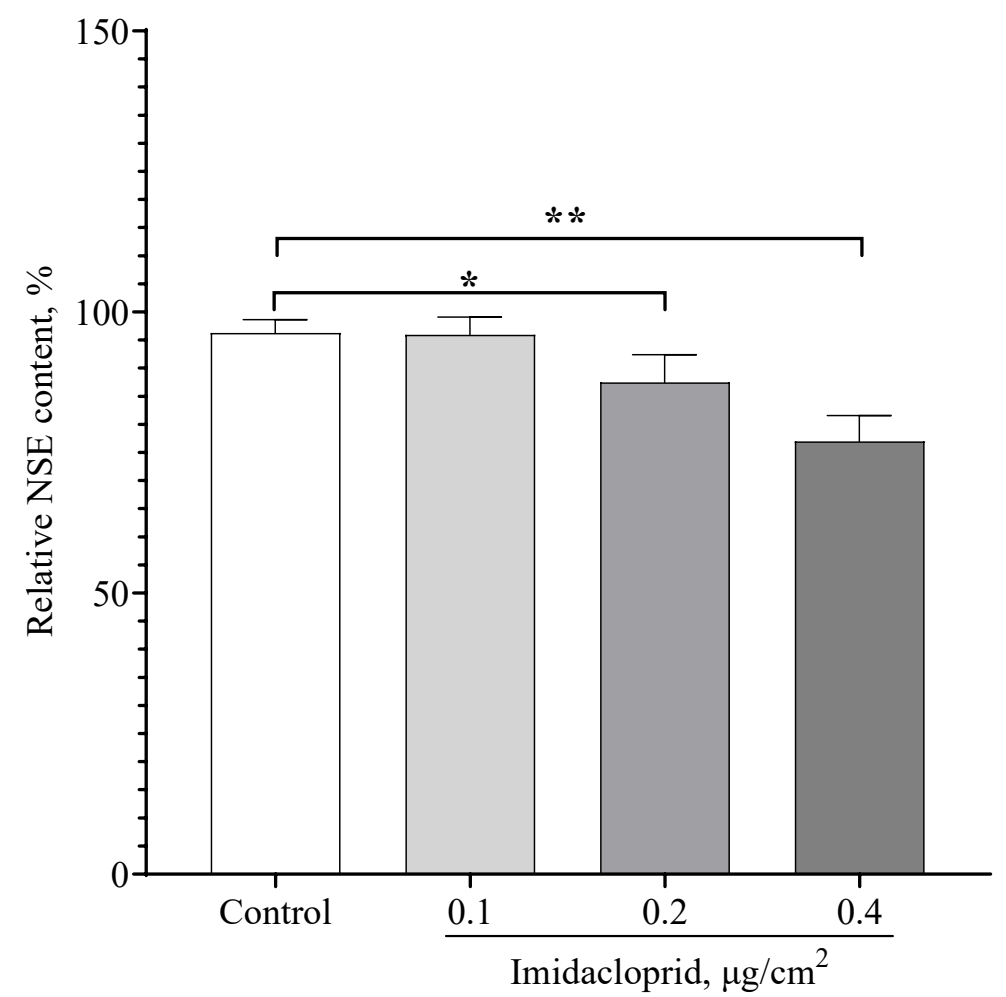

Figure 3. Effect of imidacloprid (0.1, 0.2 and $0.4 \mu \mathrm{g}$ per $1 \mathrm{~cm}^{2}$ of contact paper) on neuron-specific enolase (NSE) expression in the treated earthworms; ${ }^{*} \mathrm{p}<0.05, * * \mathrm{p}<0.01$

(compared with the control group).

The most representable data of NSE western blot results presented in a Figure 4.

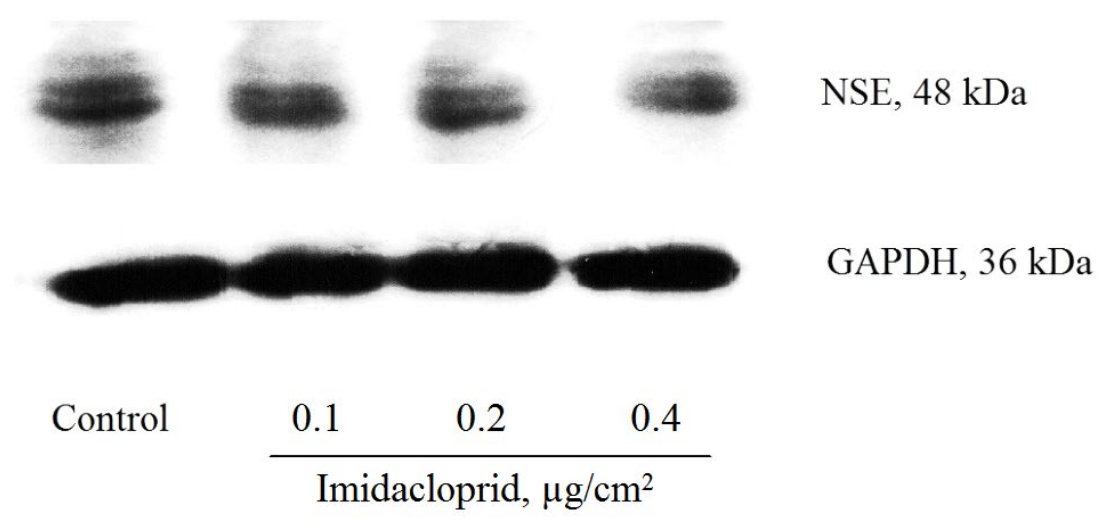

Figure 4. The western blot results of NSE detection.

Taking together the presented results, imidacloprid cytotoxicity is accompanied by the dosedependent increase of ROS and LPO levels as well as decrease of NSE.

\section{Discussion}

Presented research results support the hypothesis that low doses of imidacloprid have both cytotoxic and neurotoxic effects connected with the oxidative stress generation and the neuronspecific enolase decline in earthworms E. fetida.

The oxidative stress is the most widespread abnormality caused by xenobiotics and environmental contaminants. We studied a level of reactive oxygen species, which are the initiators of oxidizing processes. We have found the dose-dependent increase of ROS level in E. fetida caused by the exposure to low doses of imidacloprid. According to the obtained data, all of the studied low doses $\left(0.1-0.4 \mu \mathrm{g} / \mathrm{cm}^{2}\right)$ stimulated the increase of oxidative stress indices. Observed in 
our study oxidative stress is agreed with the most of literature data on general oxidative consequences of sub-chronic imidacloprid exposure $[14,46]$.

The level of malondialdehyde known as peroxidation product indicates the intensity of lipid peroxidation (LPO) processes. The LPO intensity reflects the ROS production and indicates the force of cell membranes injury. We have found significant increase of LPO levels in E. fetida caused by the exposure to low doses of imidacloprid. It should entail degenerative processes in different earthworm tissues including nervous one.

In the present study, we have detected the expression of NSE considering it as a biomarker of neuronal integrity. We have found the modulation of NSE expression under imidacloprid low doses that is undoubted evidence of imidacloprid-caused neurotoxicity in the earthworm nervous system. The neuron-specific enolase reactivity in earthworms demonstrates the functional activity in this phylogenetically ancient invertebrate species. The nervous system maintains the viability of the whole organism as well as particular organs. Observed in our study NSE decrease substantiates that imidacloprid induces neuronal disruption and possible neuronal dysfunction in earthworms, as it was reported for other animal groups $[4,33]$. The NSE alteration confirms the neuronal injury under influence of imidacloprid. Thus, cytotoxicity of imidacloprid is related to its direct effect on neuronal cells and indirect detrimental effect on the dysregulation signaling pathways in earthworm E. fetida. The revealed suppression of the NSE expression in E. fetida is presented for the first time. Obtained data on imidacloprid neurotoxicity for the earthworm nervous system may be fruitful for the assessment of the imidacloprid application risk in different soil ecosystems.

\section{Conclusions}

Low doses of imidacloprid affect the earthworm Eisenia fetida by generating oxidative stress and lipid peroxidation, which are associated with the increase in the neuron-specific enolase expression. Thus, the imidacloprid-caused cytotoxicity is accompanied by neuronal damage and the neurotoxicity could be recognized as an individual scenario of the total imidacloprid cytotoxicity. Further study is required to clarify the harmful effect of imidacloprid on the earthworm as an important component of soil biota.

\section{Conflict of Interest}

Authors declare no personal circumstances or interests that may be perceived as inappropriately influencing the representation or interpretation of reported research results.

\section{Acknowledgments}

The authors deeply acknowledge assistance from Dr. Anna Hahut for her substantial support in the methodical part of the research. The study was funded by the Ministry of Education and Science of Ukraine (Project No 0120U102258).

\section{References}

[1] E.E. Ewere et al., Uptake, depuration and sublethal effects of the neonicotinoid, imidacloprid, exposure in Sydney rock oysters, Chemosphere. 230 (2019) 1-13.

[2] E.A.D. Mitchell et al., A worldwide survey of neonicotinoids in honey, Science (New York, N.Y.). 358(6359) (2017) 109-111.

[3] Ph. Wexler (Ed.) Encyclopedia of Toxicology, Academic Press, Elsevier Inc., 2014.

[4] I.Y. Abd-Elhakim et al., Imidacloprid impacts on neurobehavioral performance, oxidative stress, and apoptotic events in the brain of adolescent and adult rats, Journal of Agricultural and Food Chemistry. 66(51) (2018) 13513-13524. 
[5] L. Donnarumma et al., Preliminary study on persistence in soil and residues in maize of imidacloprid, Journal of Environmental Science and Health, Part B. 46(6) (2011) 469-472.

[6] $\mathrm{P}$. $\mathrm{Wu}$ et al., The imidacloprid remediation, soil fertility enhancement and microbial community change in soil by Rhodopseudomonas capsulata using effluent as carbon source, Environmental Pollution. 267 (2020) 114254.

[7] X. Wang et al., Multi-level ecotoxicological effects of imidacloprid on earthworm (Eisenia fetida), Chemosphere. 219 (2019) 923-932.

[8] J.D. Knoepp et al., Imidacloprid movement in soils and impacts on soil microarthropods in southern Appalachian eastern hemlock stands, Journal of Environmental Quality. 41(2) (2012) 469478

[9] T. Bhadauria, K.G. Saxena, Role of earthworms in soil fertility maintenance through the production of biogenic structures, Applied and Environmental Soil Science. 2010 (2009) 816073.

[10] W. Ge et al., Oxidative stress and DNA damage induced by imidacloprid in zebrafish (Danio rerio), Journal of Agricultural and Food Chemistry. 63(6) (2015) 1856-1862.

[11] N. Malhotra et al., Physiological effects of neonicotinoid insecticides on non-target aquatic animals - an updated review, International Journal of Molecular Sciences. 22(17) (2021) 9591.

[12] R. Miglani, S.S. Bisht, World of earthworms with pesticides and insecticides. Interdisciplinary Toxicology, 12(2) (2019) 71-82.

[13] C. Pelosi et al., Pesticides and earthworms. A review, Agronomy for Sustainable Development. 34 (2014) 199-228.

[14] Q. Zhang, B. Zhang, C. Wang, Ecotoxicological effects on the earthworm Eisenia fetida following exposure to soil contaminated with imidacloprid, Environmental Science and Pollution Research International. 21(21) (2014) 12345-12353.

[15] B. Bradford et al., Neonicotinoid-containing insecticide disruption of growth, locomotion, and fertility in Caenorhabditis elegans, PLoS One. 15(9) (2020) e0238637.

[16] D. Goulson, An overview of the environmental risks posed by neonicotinoid insecticides, Journal of Applied Ecology. 50(4) (2013) 977-987.

[17] Y. Capowiez, A. Bérard, Assessment of the effects of imidacloprid on the behavior of two earthworm species (Aporrectodea nocturna and Allolobophora icterica) using 2D terraria, Ecotoxicology and Environmental Safety. 64(2) (2006) 198-206.

[18] F. Chevillot et al., Selective bioaccumulation of neonicotinoids and sub-lethal effects in the earthworm Eisenia andrei exposed to environmental concentrations in an artificial soil, Chemosphere. 186 (2017) 839-847.

[19] C. de Lima e Silva at al., Comparative toxicity of imidacloprid and thiacloprid to different species of soil invertebrates, Ecotoxicology. 26(4) (2017) 555-564.

[20] C. de Lima e Silva at al., Toxicity in neonicotinoids to Folsima candida and Eisenia andrei, Environmental Toxicology and Chemistry. 39(3) (2020) 548-555.

[21] V.D. Dani et al., Comparison of metabolomic responses of earthworms to sub-lethal imidacloprid exposure in contact and soil tests, Environmental Science and Pollution Research International. 26(18) (2019) 18846-18855.

[22] H. Zhang et al., Differences in kinetic metabolomics in Eisenia fetida under single and dual exposure of imidacloprid and dinotefuran at environmentally relevant concentrations, Journal of Hazardous Materials. 417 (2021) 126001. 
[23] V.S. Nedzvetsky et al., Soluble curcumin ameliorates motility, adhesiveness and abrogate parthanatos in cadmium-exposed retinal pigment epithelial cells, Biosystems Diversity. 29(3) (2021) 235-243.

[24] M. Rusz et al., Morpho-metabotyping the oxidative stress response, Scientific Reports. 11 (2021) 15471.

[25] Y. Zang et al., Genotoxicity of two novel pesticides for the earthworm, Eisenia fetida, Environmental Pollution. 108(2) (2000) 271-278.

[26] T. Liu et al., Biochemical and genetic toxicity of dinotefuran on earthworms (Eisenia fetida). Chemosphere. 176 (2017) 156-164.

[27] Y. Liu et al., Thiamethoxam exposure induces endoplasmic reticulum stress and affects ovarian function and oocyte development in mice, Journal of Agricultural and Food Chemistry. 69(6) (2021) 1942-1952.

[28] M. Liu et al., From the cover: exposing imidacloprid interferes with neurogenesis through impacting on chick neural tube cell survival, Toxicological Sciences. 153(1) (2016) 137-148.

[29] R. Mesnage et al., Evaluation of neonicotinoid insecticides for oestrogenic, thyroidogenic and adipogenic activity reveals imidacloprid causes lipid accumulation, Journal of Applied Toxicology. 38(12) (2018) 1483-1491.

[30] V.S. Nedzvetsky et al., Low doses of imidacloprid induce disruption of intercellular adhesion and initiate proinflammatory changes in Caco-2 cells, Regulatory Mechanisms in Biosystems. 12(3) (2021) 430-437.

[31] J. Kim et al., Imidacloprid, a neonicotinoid insecticide, induces insulin resistance, The Journal of Toxicological Sciences. 38(5) (2013) 655-660.

[32] M. Abou-Donia et al., Imidacloprid induces neurobehavioral deficits and increases expression of glial fibrillary acidic protein in the motor cortex and hippocampus in offspring rats following in utero exposure, Journal of Toxicology and Environmental Health, Part A. 71(2) (2008) 119-130.

[33] S.M. Rawi, A.S. Al-Logmani, R.Z. Hamza, Neurological alterations induced by formulated imidacloprid toxicity in Japanese quails, Metabolic Brain Disease. 34(2) (2019) 443-450.

[34] V.Ya. Gasso et al., Biomarkers of the influence of pyrethroids and neonicotinoids on amphibian larvae, Ecology and Noospherology. 31(1) (2020) 46-51.

[35] V.Y. Gasso et al., Local industrial pollution induces astrocyte cytoskeleton rearrangement in the dice snake brain: GFAP as a biomarker, Biosystems Diversity. 28(3) (2020) 250-256.

[36] M. Kirici et al., Sublethal doses of copper sulphate initiate deregulation of glial cytoskeleton, NF-kappa B and PARP expression in Capoeta umbla brain tissue, Regulatory Mechanisms in Biosystems. 10(1) (2019) 103-110.

[37] V.S. Nedzvetsky et al., Influence of the insecticide $\lambda$-cyhalothrin on oxidative stress and expression of replicative protein A in the brain of fish, Agrology. 3(4) 2020 214-218.

[38] R. Cliff et al., Effect of diesel exhaust inhalation on blood markers of inflammation and neurotoxicity: a controlled, blinded crossover study, Inhalation Toxicology. 28(3) (2016) 145-153.

[39] A. Ramirez-Celis et al., Peptides of neuron specific enolase as potential ASD biomarkers: from discovery to epitope mapping, Brain, Behavior, and Immunity. 84 (2020) 200-208.

[40] M.A. Isgrò, P. Bottoni, R. Scatena, Neuron-specific enolase as a biomarker: biochemical and clinical aspects, Advances in Experimental Medicine and Biology. 867 (2015) 125-143. 
[41] M.Z. Jeddi et al., Towards a systematic use of effect biomarkers in population and occupational biomonitoring, Environment International. 146 (2021) 106257.

[42] V.B. Archibong et al., The effect of codeine administration on oxidative stress biomarkers and the expression of the neuron-specific enolase in the brain of Wistar rats, NaunynSchmiedeberg's Archives of Pharmacology. 394(8) (2021) 1665-1673.

[43] OECD, Test No. 207: Earthworm, Acute Toxicity Tests, OECD Guidelines for the Testing of Chemicals, Section 2, OECD Publishing, Paris, 1984.

[44] H. Ohkawa, N. Ohishi, K. Yagi, Assay for lipid peroxides in animal tissues by thiobarbituric acid reaction, Analytical Biochemistry. 95(2) (1979) 351-358.

[45] T.S. Hnasko, R.M. Hnasko, The Western blot, Methods in Molecular Biology. 1318 (2015) $87-96$.

[46] J. Wang et al., DNA damage and oxidative stress induced by imidacloprid exposure in the earthworm Eisenia fetida, Chemosphere. 144 (2016) 510-517. 